\title{
El proyecto de vida como método didáctico para el desarrollo de competencias literarias1
}

\section{The Life Project as a Didactic Method for the Development of Literary Competences}

DOI: http://dx.doi.org/10.17981/cultedusoc.9.3.2018.12

Artículo de investigación. Fecha de recepción: 15/06/2018. Fecha de aceptación: 27/11/2018

Martha Castro-Colón";

Onilda Escorcia-Pacheco; Sinivaldo Montenegro y Sonia Luz-Ospino ${ }^{3}$

Institución Educativa Departamental Juan Manuel Rudas (Colombia)

hormiga777@hotmail.com

Para citar este artículo:

Castro-Colón. M., Escorcia-Pacheco. O., Montenegro, S. y Luz-Ospino, S. (2018). El proyecto de vida como método didáctico para el desarrollo de competencias literarias. Cultura. Educación y Sociedad 9(3), 99-104. DOI: http://dx.doi.org/10.17981/cultedusoc.9.3.2018.12

\section{Resumen}

El ser humano desde el desarrollo inicial de su personalidad tiende a accionar de acuerdo a sus motivaciones e intereses, razón por la cual se plantea metas a corto, mediano y largo plazo. Es por esto que los proyectos de vida se han convertido en una herramienta que permite trazar rutas hacia los logros que los seres humanos quieren alcanzar en plazos. El presente estudio tuvo como objetivo promover el proyecto de vida como método didáctico para el desarrollo de competencias literarias. La población participante fueron 30 estudiantes de la Institución Educativa Departamental Juan Manuel Rudas., se llevó a cabo desde una mirada cualitativa, de alcance descriptivo, con un diseño de campo, siguiente la ruta de la IEP. Los resultados permiten dejan ver que los estudiantes se motivan a participa en las actividades en donde les permiten mostrar cuáles son sus planes, que desean para el futuro, como van hacer para lograrlo, y se ha notado el mayor interés frente a las actividades educativas.

Palabras clave: Educación didáctica, proyecto de vida, competencias literarias.

\section{Abstract}

The human being from the initial development of his personality tends to act according to their motivations and interests, which is why he considers short, medium and long term goals. This is why life projects have become a tool that allows us to trace routes towards the achievements that human beings want to achieve in terms. The objective of this study was to promote the life project as a didactic method for the development of literary competences. The participating population was 30 students from the Departmental Educational Institution Juan Manuel Rudas., It was carried out from a qualitative perspective, with a descriptive scope, with a field design. The results allow to see that students are motivated to participate in activities where they can show what their plans are, what they want for the future, how they are going to do it, and the greatest interest in educational activities has been noticed.

Keywords: Didactic education, life project, literary competitions.

1 Este articulo ha sido derivado del programa de fortalecimiento de la cultura ciudadana y democrática CT+I a través de la IEP apoya en TIC en el Departamento de Magdalena: CICLON

2 Líder del grupo de investigación: Grupo de investigación "Los Renovadores".

3 Docentes de la institución Educativa Departamental Juan Manuel Rudas y del grupo de investigación “Los Renovadores”.

- The author; licensee Universidad de la Costa - CUC.

Cultura, Educación y Sociedad vol. 9 no. 3, pp. 99-104. Diciembre, 2018

Barranquilla. ISSN 2389-7724 Online 


\section{Introducción}

Remolino es un pueblo ribereño, bañado por el caudaloso rio Magdalena, ubicado al noroccidente del Departamento del Magdalena. Las familias de esta población son de escasos recursos económicos, la mayoría solo labora en épocas de cosecha, otros se trasladan a trabajar al Departamento del Atlántico, dejando a sus hijos a cargo de familiares. Situación que genera que los niños, adolescentes y jóvenes no aprovechen su tiempo libre al estar mucho tiempo en la calle luego de terminar la jornada escolar, de igual forma el nivel educativo de los padres de familia es bajo.

Todo esto llevó a detectar porqué la falencia en el estudio y en su comportamiento, al carecer de un plan de vida que les permita establecer metas para logar sus sueños en el presente y en el futuro; de esta manera surge la idea implementar un proyecto de vida en ellos, que busque la transformación del ser asumiendo responsabilidades y el deseo de salir adelante.

Los estudiantes de la Institución Educativa Departamental Juan Manuel Rudas muestran poco interés en sus labores académicas, es decir, su actitud hacia el estudio es mas de apatía, cansancio e inclusive rechazo; sumado a esto los padres de familia no son un modelo a seguir, por las diferentes ocupaciones diarias de las cabezas del hogar demuestran menos compromiso con relación a las obligaciones estudiantiles de sus hijos. Culturalmente hay pocos patrones del medio ambiente en el que se desenvuelven que aumenten y motiven la participación activa y permanente de la escuela.

Cultura, son todas aquellas configuraciones que realizan los habitantes de un pueblo, con base en sus manifestaciones sociales, dinámicas cotidianas y todo aquello que la naturaleza les haya provisto, tanto de manera individual como colectiva (Rogers,
1996). En esos procesos de intercambio, el hombre crea religión, mitos, arte, ciencia, tecnología, moral, costumbres y un sinnúmero de formas de pensar, actuar y construir la historia de la comunidad (Arrieta, 2016); los cuales son transmitidos de generación en generación y contribueyen a mantener la identidad y la forma de vida de los pueblos (Camps, 1994).

\section{Importancia de la familia y el contexto en el desarrollo del proyecto de vida}

Bowlby (1986) en sus postulados estableció que el apego es el vínculo emocional que establece el niño hacia sus padres y/o cuidadores, que le permite desarrollar seguridad emocional y el pleno desarrollo de su personalidad; en donde influye también como factor prioritario el ambiente donde se desenvuelve el niño, es por ello que la familia y el contexto son fuente primaria de socialización. Desde esta perspectiva, la función socializadora de la familia es única, pues es allí, en ese ámbito, en donde las personas tienen la oportunidad de estructurarse como sujetos, de reconocerse a través de otros y como parte de algo, un algo que le permite ser de una manera particular (Donati, 2003; Pérez Adán, 2006; Bowlby 1986).

Bowlby (1980) además, identifica modelos de transmisión del apego, que se determinan y transmiten de generación en generación, mediante "estados de la mente" y define que tales modelos se comunican de padre a hijo(a) mediante patrones de conducta y principalmente en situaciones estresantes, que es cuando se activa el sistema de apego. La familia es decisiva para el aprendizaje de valores éticos, la educación de los niños, niñas y adolescentes, la formación de la inteligencia emocional, la construcción de identidades, la expresión del amor y la espiritualidad (Kliksberg, 2008). Los aprendizajes obtenidos en la familia, contribuyen de manera positiva 
o negativa a las relaciones sociales fuera de ella.

Arriagada (2009) sostiene que la familia al igual que otras instituciones sociales, también experimenta transformaciones, producto de los cambios sociales, económicos e históricos como la emergencia y coexistencia de distintas formas de constitución de la familia, nuevos modos de relación entre sus miembros y cambios en los roles y sentidos. Sin embargo, se mantienen las desigualdades de género y persistencia de la violencia intrafamiliar y escolar (Herrera-Mendoza y Rico, 2014).

\section{Proyecto de vida}

A nivel teórico, el proyecto de vida no siempre encaja con las acciones prácticas de los seres humanos, y es entendible en el sentido que la vida no es controlable al 100\%. Lo importante en el proyecto de vida es identificar escenarios en los que la persona se ubique donde desea estar y se sienta satisfecho con lo que ha logrado en la vida. Si esto no ocurre, es posible que el individuo experimente tristeza, insatisfacción y la sensación de no haber aprovechado bien el tiempo (Rovira, 1996).

El desarrollo del proyecto de vida se hace de manera individual, aunque existen momentos en los que los proyectos de vida de varos individuos confluyen entre sí, como es el caso de conformación de una pareja u hogar (Zamora, 2014). Si los proyectos de vida de varias personas no son compatibles, es necesario conciliar, con el fin de definir puntos de encuentro a partir de los cuales sea posible establecer nexos de unión entre las partes (Santana, 2012).

En los espacios académicos, los proyectos de vida son empleados como escenarios en los que se lleva a los estudiantes a reflexionar sobre lo que han realizado y lo que esperan en su futuro, con base en las dimensiones en las que se desenvuelven. En este sentido, es posible hablar de la socialización de conocimientos entre los estudiantes, en los que es posible, no sólo integrar saberes específicos desde las asignaturas que se imparten, sino que además, es posible afianzar competencias en lo que respeta al "saber ser" y "saber hacer". Con el uso de la didáctica en este proceso, se establecen métodos, técnicas y estrategias que contribuyen al mejoramiento del aprendizaje y al desarrollo de mejores reflexiones sobre el presente y futuro de los estudiantes.

\section{Metodología}

Se realizó un estudio de tipo cualitativo, siendo ésta la que busca conocer e interpretar la realidad de los participantes a través de sus propias experiencias, entregando una información subjetiva del fenómeno de estudio, el alcance es descriptivo, con un diseño de campo.

\section{Participantes}

Durante el estudio participaron 30 estudiantes distribuidos en los cursos pertenecientes a la educación básica primaria, con edades que oscilaron entre los 9 y 11 años de la Institución Educativa Departamental Juan Manuel Rudas.

\section{Técnicas e Instrumentos}

Como soporte instrumental para el desarrollo del abordaje metodológico se contó con el diseño de formatos semi-estructurados para el registro de la información derivada de las actividades de investigación activa y participativa. Los formatos correspondieron a las actividades grupales de la tarjeta viajera, sesiones focales de padres de familia referente al proyecto de vida se tomaron registro de información por medio observación directa, diarios de campo que se tomaron en medio de los desarrollado. 


\section{Procedimiento}

La propuesta se desarrolló siguiendo la ruta de la IEP definida por los momentos, evidenciado en las actividades grupales como lo son: la libreta viajera, encuentros con padres de familia, y el proyecto de vida, donde se trabajaron las competencias ciudadanas y puedan compartir sus experiencias de vida a partir de una investigación sobre ellos mismos y su entorno.

\section{Resultados}

Con el fin hacer de estas actividades mucho más lúdicas e interactivas se realizaron diferentes actividades soportadas con el uso de las TIC (ejercicios con texto en internet, videos en YouTube y documentos sobre las redes sociales), promoviendo a su vez el desarrollo de prácticas de uso adecuado de las TIC. (Ver tabla 1)

TABLA 1

Resultados desde las actividades literarias
A partir del análisis de los hallazgos observados durante el desarrollo de la investigación del proyecto en la IED, se puede afirmar que resultó ser una estrategia significativa para el fomento del aprendizaje; siendo los estudiantes los que manifiestan un apego por aprender, pues es de su interés participar en las actividades lúdicas de acuerdo a los intereses y proyectos personales que ellos tienen.

Adicional, se identificó la necesidad de seguir fomentando estrategias de cambio que se orienten a fortalecer su proyecto de vida, a partir de las apreciaciones y recomendaciones de algunos autores que hacen referencia a la falta de hábito de la lectura, el abandono de los textos literarios, la mala ortografía, la falta de cultura, la dificultad de crear argumentos para defender posiciones y la pérdida de las habilidades comunicativas, en congruencia con los aportes de Montenegro, Blanco \& Cortés (2013).

\begin{tabular}{|c|c|c|}
\hline Actividad & Acciones / Resultados & Recursos \\
\hline Conocemos la IEP & Talleres sobre la IEP y el uso dentro de los proyectos de vida. & \multirow{8}{*}{$\begin{array}{l}\text { Computadores, } \\
\text { tabletas } \\
\text { Papeles, } \\
\text { colores, lápices, } \\
\text { marcadores } \\
\text { Cartulina, } \\
\text { colores, dulces, } \\
\text { premios, } \\
\text { marcadores, } \\
\text { cartón, hojas } \\
\text { de block }\end{array}$} \\
\hline $\begin{array}{l}\text { Jugando con las } \\
\text { Tecnologías }\end{array}$ & $\begin{array}{l}\text {-Talleres sobre el uso de las redes sociales. } \\
\text {-Actividades de videos en YouTube y lecturas. }\end{array}$ & \\
\hline $\begin{array}{l}\text { ¿Quién soy y cómo } \\
\text { me proyecto? }\end{array}$ & $\begin{array}{l}\text { Actividad en donde en cinco líneas deben resumir los aspecto más } \\
\text { importantes de su vida. }\end{array}$ & \\
\hline $\begin{array}{l}\text { Nuestra } \\
\text { autoestima }\end{array}$ & $\begin{array}{l}\text { Encuentros con padres de familias en los cuales se adelantó } \\
\text { la promoción del desarrollo afectivo y el mejoramiento de las } \\
\text { relaciones familiares, lo que incidió en el incremento de su } \\
\text { autoestima. }\end{array}$ & \\
\hline La libreta viajera & $\begin{array}{l}\text { Una actividad en la que los estudiantes y sus padres participaron } \\
\text { en la elaboración de su proyecto de vida. }\end{array}$ & \\
\hline $\begin{array}{l}\text { Mi proyecto de } \\
\text { vida }\end{array}$ & $\begin{array}{l}\text { Jornadas con los niños y jóvenes que permitieron conocer quiénes } \\
\text { son, que quieren y como se proyectan en su futuro en compañía de } \\
\text { sus seres más allegados. }\end{array}$ & \\
\hline $\begin{array}{l}\text { Descubro mi } \\
\text { entorno } \\
\text { (Reconocimiento } \\
\text { del contexto). }\end{array}$ & $\begin{array}{l}\text { Actividad donde se le presentó un texto sobre alguna situación } \\
\text { cotidiana, para que fuera interpretada por los estudiantes desde } \\
\text { su punto de vista. Esta actividad incrementó los niveles de } \\
\text { análisis socio-crítico }\end{array}$ & \\
\hline $\begin{array}{l}\text { Dibujo y creo una } \\
\text { historia }\end{array}$ & $\begin{array}{l}\text { Cada compañero dibujaba una imagen, luego se rotaron los } \\
\text { dibujos y cada uno de los estudiantes creó una historia a partir de } \\
\text { los dibujos, incrementando los niveles de aprendizaje colaborativo } \\
\text { y el desarrollo de la creatividad. }\end{array}$ & \\
\hline
\end{tabular}

Fuente: elaboración propia. 


\section{Discusión}

El proyecto de vida en los estudiantes constituye una herramienta impulsadora de motivación, le permite visionarse al futuro, y le muestra alternativas para lograrlo. Los resultados dejan ver que si bien los estudiantes asumen con actitud positiva nuevas metodologías de aprendizaje a través del fortalecimiento del proyecto de vida, es necesario ahondar y fortalecer novedosas estrategias articuladas a las necesidades pedagógicas.

Los estudiantes a nivel promedio desconocen que es un proyecto de vida, y aunque participan activamente resultado complejo lograr que se cumplan los propósitos de articulación con las competencias, por lo tanto es un llamado a la comunidad docente a trabajar en nuevas y novedosas tareas de enseñanza proactiva y participativa.

\section{Referencias}

Arrieta, M. (2016). Los masones en el mundo: Geopolítica masónica. La historia de los hijos de la Luz. Córdoba: Editorial Almuzara.

Bowlby, J. (1986). Vinculos Afectivos: Formación, Desarrollo y Pérdida. Madrid: Ediciones Morata

Camps, V. (1994). Los valores de la educación. Madrid: Anaya.

Cortés-Peña OF, Pinto-Santos AR, Atrio SI. (2015). E-portafolio como herramienta construccionista del aprendizaje activo en tecnología educativa. Revista Lasallista de Investigación, 12(2). 36-44.

Cuevas, M. (2012). Ellos también tienen voz. [Tesis de master]. Universidad de Cantabria, Santander.

Domínguez, L. (2007). Proyecto de vida y valores: condiciones de la personalidad madura y saludable. Boletín electrónico de Investigación de la Asociación Oaxaqueña de Psicología, 3(1), 44-58.
Donati, P. (1999). Familias y las generaciones. Revista Desacatos. Revista de Antropología Social, (2), 27-49. Recuperado de http://www.scielo.org.mx/ pdf/desacatos/n2/n2a3.pdf

Hernández, O. (2006). Proyecto de vida y desarrollo integral humano. Rev. Internal Crecemos. (Puerto Rico), 6(1-20), 1-31.

Herrera-Mendoza, K., y Rico, R. (2014). El Clima Escolar Como Elemento Fundamental de la Convivencia en la Escuela. Revista escenarios, 12(2). 7-18.

Marín, F., Niebles, M., Sarmiento, M. y Valbuena, S. (2017). Mediación de las tecnologías de la información en la comprensión lectora para la resolución de problemas aritméticos de enunciado verbal. Espacios, 38(20), 20-25.

Martínez, M., Pérez, W. y Solano, D. (2011). Impacto de los medios masivos de comunicación en la dinámica familiar. Cultura Educación y Sociedad, 2(1), 111-118.

Membiela, P. (2002). ¿Qué es el movimiento educativo ciencia, tecnología, sociedad? Enseñanza de las ciencias desde la perspectiva ciencia-tecnología-sociedad: formación científica para la ciudadanía, 89, 91.

Montenegro, M., Blanco, P. y Cortés, O. (2013). Papel de las habilidades metalingüísticas en los procesos de lectura y escritura en la educación superior. Escenarios, 11(2), 82-86.

Rogers, C., Freiberg, H. y Soler, S. (1996). Libertad y creatividad en la educación. Buenos Aires: Paidós.

Rovira, J. (1996). La construcción de la personalidad moral (Vol. 30). Grupo Planeta (GBS).

Sánchez, G., Aguirre, M., Solano, N. y Viveros, E. (2015). Sobre la dinámica familiar. Revisión documental. Cultura Educación y Sociedad, 6(2), 117138. 
Vargas, R. (2005). Proyecto de vida y planeamiento estratégico personal. Lima: Trepaud.
Zamora, Z. (2014). Estudio exploratorio sobre el proyecto de vida en el adulto mayor. Psicología y salud, 16(1), 103-110. 